\title{
Weaning from inhaled corticosteroids in COPD: the evidence
}

\author{
Samy Suissa ${ }^{1}$ and Andrea Rossi ${ }^{2}$
}

Affiliations: 'Department of Epidemiology and Biostatistics, Centre for Clinical Epidemiology, Lady Davis Institute - Jewish General Hospital, McGill University, Montreal, QC, Canada. ${ }^{2}$ Pulmonary Unit, Department of Medicine, University and General Hospital (AOUI) of Verona, Verona, Italy.

Correspondence: Samy Suissa, Centre for Clinical Epidemiology, Jewish General Hospital, 3755 Cote Ste-Catherine, H-461, Montreal, Québec, H3T 1E2, Canada. E-mail: samy.suissaAmcgill.ca

0 $@$ ERSpublications

Much inappropriate use of inhaled corticosteroids in COPD can be safely replaced with longacting bronchodilators http://ow.ly/QvMRd

The use of inhaled corticosteroids (ICS) in the treatment of chronic obstructive pulmonary disease (COPD) has been widely debated [1-3]. Treatment guidelines have suggested that their prescription be limited to COPD patients with severe airflow limitation at high risk of exacerbations, who remain symptomatic after regular use of one or two long-acting bronchodilators [4]. Nevertheless, ICS have been used widely, with recent trials observing that over $70 \%$ of COPD patients were treated with ICS at the time of enrolment. Evidence is mounting that such extensive use of ICS is discrepant with COPD treatment guidelines and may be inappropriate in a subset of these users $[5,6]$.

Consequently, four randomised trials have been conducted to assess the effects of ICS discontinuation in COPD patients, producing somewhat mixed results [7-10]. In this editorial, we discuss and address their methodological particularities, focussing on the major end-points of COPD exacerbation and lung function.

\section{The randomised controlled trials}

The four randomised trials that evaluated the effect of discontinuing the ICS component of different treatments involved ICS in single, double or triple therapy (figure 1). Table 1 describes some design characteristics of these trials and provides data on the end-points.

The COPE trial randomised, after 4 months of treatment with fluticasone propionate $(1000 \mu \mathrm{g}$ per day), 244 patients to either continue or to discontinue (placebo) the ICS [7]. During the 6-month follow-up, $57 \%$ of patients who discontinued ICS had an exacerbation compared with $47 \%$ of those who continued (hazard ratio $1.5,95 \%$ CI 1.1 to 2.1 ), with this difference concentrated in the first 50 days of follow-up. At 6 months, the relative loss in forced expiratory volume in $1 \mathrm{~s}$ (FEV1) with discontinuation was $38 \mathrm{~mL}$ ( $95 \% \mathrm{CI}-2$ to $80 \mathrm{~mL}$ ).

The two subsequent trials involved discontinuation among patients given an ICS/long-acting $\beta_{2}$-agonist (LABA) combination, replacing it with a LABA only. The COSMIC trial, which involved 373 patients with COPD who, after a 3-month run-in treatment period with fluticasone propionate $(1000 \mu \mathrm{g}$ per day) combined with salmeterol, were randomised to continue or to discontinue the ICS component, replaced by salmeterol only [8]. During 1-year follow-up, the rate ratio of moderate or severe exacerbation associated with ICS discontinuation was $1.2(95 \%$ CI 0.9 to $1.5, \mathrm{p}=0.15)$ while for mild exacerbations, it was $2.0(95 \%$ CI 1.1 to $3.5, \mathrm{p}=0.02$ ). Of note, the Poisson regression model used in this analysis was not adjusted for between-patient variability, thus underestimating the p-values [11, 12]. At 12 months, the relative loss in FEV1 with discontinuation was significant at $50 \mathrm{~mL}(95 \% \mathrm{CI} 10$ to $100 \mathrm{~mL})$.

Received: Feb 172015 | Accepted after revision: June 162015

Conflict of interest: Disclosures can be found alongside the online version of this article at erj.ersjournals.com

Copyright OERS 2015 
FIGURE 1 Randomised controlled trial design. ICS: inhaled corticosteroids; LABA: long-acting

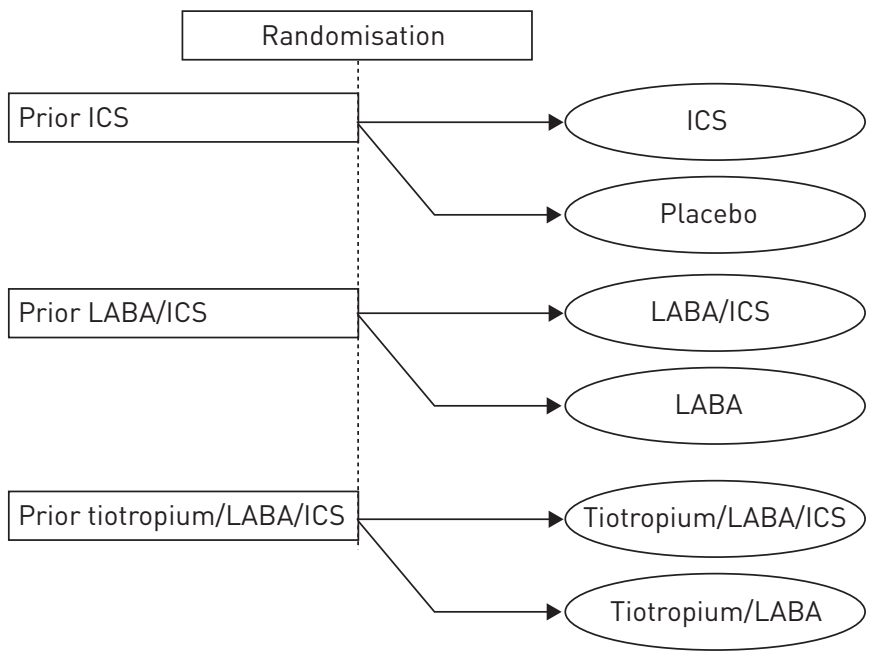
$\beta_{2}$-agonist.

The INSTEAD trial investigated ICS discontinuation in patients at low risk of COPD exacerbation, namely with no COPD exacerbations for a year, and already treated with salmeterol/fluticasone combination (SFC) for $\geqslant 3$ months [9]. The trial randomised 581 such patients to either continue their SFC treatment or to switch to monotherapy with indacaterol, a once-daily LABA bronchodilator. During the 6-month follow-up, the rate ratio of any mild, moderate or severe exacerbation associated with ICS discontinuation was 0.86 (95\% CI 0.62 to $1.20, \mathrm{p}=0.37$ ). The hazard ratio of the first moderate or severe exacerbation associated with ICS discontinuation was $0.80(\mathrm{p}=0.26)$. At 6 months, the relative FEV1 loss with discontinuation was $9 \mathrm{~mL}(95 \% \mathrm{CI}-26$ to $45 \mathrm{~mL})$.

The fourth trial is WISDOM, which investigated ICS discontinuation in patients given triple therapy consisting of tiotropium, salmeterol and fluticasone propionate [10]. The 2485 study patients, who had a history of exacerbation of COPD and received the triple therapy for a 6-week run-in period, were randomised to continue triple therapy or to wean off fluticasone gradually over a 12 -week period. During the 12-month follow-up, the hazard ratio of the first moderate or severe exacerbation associated with ICS discontinuation was 1.06 (95\% CI 0.94 to 1.19), while for the first severe exacerbation it was 1.20 (95\% CI 0.98 to 1.48). At 12 months, there was a significant FEV1 relative loss with discontinuation of $43 \mathrm{~mL}$ (95\% CI 17 to $69 \mathrm{~mL}$ ).

TABLE 1 Randomised trials of the effect of inhaled corticosteroid (ICS) discontinuation in chronic obstructive pulmonary disease (COPD) on the relative risk of COPD exacerbation and on the relative loss of forced expiratory volume in $1 \mathrm{~s}$ (FEV1)

\begin{tabular}{|c|c|c|c|c|}
\hline & COPE & COSMIC & INSTEAD & WISDOM \\
\hline Mean age years & 64 & 64 & 66 & 64 \\
\hline Prior exacerbations per year & 1.3 & $\geqslant 2$ & 0 & $\geqslant 1$ \\
\hline Prior ICS use \% & 83 & 86 & 100 & 70 \\
\hline Baseline FEV 1 \% predicted & 57 & 48 & 64 & 34 \\
\hline Initial ICS-based therapy & ICS & LABA/ICS & LABA/ICS & Tiotropium/LABA/ICS \\
\hline ICS-based run-in duration months & 4 & 3 & $>3.5$ & 1.5 \\
\hline Study follow-up months & 6 & 12 & 6 & 12 \\
\hline \multicolumn{5}{|l|}{ Effects of ICS discontinuation } \\
\hline \multicolumn{5}{|l|}{ Relative risk of exacerbation $(95 \% \mathrm{CI})$} \\
\hline Any & $1.5(1.1$ to 2.1$)$ & & $0.9(0.6$ to 1.2$)$ & \\
\hline Mild & & $2.0(1.1 \text { to } 3.5)^{\#}$ & & \\
\hline Moderate/severe & & $1.2(0.9 \text { to } 1.5)^{\#}$ & $0.8(0.5 \text { to } 1.2)^{9}$ & 1.1 (0.9 to 1.2$)$ \\
\hline Severe & & & & $1.2(1.0$ to 1.5$)$ \\
\hline FEV 1 loss $\mathrm{mL}$ mean $(95 \% \mathrm{CI})$ & 38 (-2 to 80$)$ & $50(10$ to 100$)$ & $9(-26$ to 45$)$ & $43(17 \text { to } 69)^{\pi}$ \\
\hline
\end{tabular}

LABA: long-acting $\beta_{2}$-agonist. " : rate ratio estimated by Poisson regression model not adjusted for between-patient variability, thus leading to artificially narrower confidence intervals; ${ }^{\text {n: }}$ 95\% confidence interval extrapolated from reported $p$-values. 


\section{Risk reductions}

No study has specifically evaluated the impact of ICS discontinuation on the reduction of adverse outcomes, which include reducing the elevated risks of pneumonia, cataracts, fractures, tuberculosis and diabetes, known to be associated with ICS use [3]. However, two of the trials provided data on pneumonia as an adverse event (table 2). In the INSTEAD trial, there were two pneumonia events in the group that continued their ICS treatment compared with 0 events in the group that switched to indacaterol monotherapy over the 6-month follow-up [9]. In the WISDOM trial, pneumonia was present in $5.8 \%$ of patients in the group that continued ICS treatment compared with $5.5 \%$ in the group that discontinued over the 12-month follow-up [10].

\section{Discussion}

With the now recognised widespread overuse of ICS in the treatment of COPD, the question of the effects of ICS discontinuation in patients whose use is inappropriate is receiving greater attention. Four trials have evaluated the effects of ICS withdrawal with somewhat inconsistent results, which may be due to their methodological particularities.

One issue is the starting study treatment from which the effect of ICS discontinuation is to be evaluated. The COPE trial downgraded from single ICS therapy to no ICS, the COSMIC and INSTEAD trials downgraded from double therapy (ICS/LABA) to LABA only, and the WISDOM trials went from triple therapy (ICS/LABA/tiotropium) to the LABA/tiotropium combination. The COPE trial, while scientifically valid, is not clinically useful as it involves exclusive treatment with ICS, which is not a recognised approach. Indeed, all guidelines recommend long-acting bronchodilators as baseline therapy, with ICS to be added if necessary; none recommends ICS monotherapy in COPD.

The more clinically relevant COSMIC, INSTEAD and WISDOM trials, which discontinued ICS from double or triple therapy, all agree that there essentially is no effect of ICS discontinuation on moderate or severe exacerbations. An exception is the significant effect for mild exacerbation in the COSMIC trial, which is likely to be a statistical artefact from the analysis not adjusted for between-patient variability. However, the significant relative loss in $\mathrm{FEV}_{1}$ of $50 \mathrm{~mL}$ in COSMIC and $43 \mathrm{~mL}$ in WISDOM are at odds with the nonsignificant loss of $9 \mathrm{~mL}$ found in INSTEAD, though its $95 \%$ confidence interval is compatible with a loss of up to $45 \mathrm{~mL}$. The fact that loss to follow-up in these trials was limited and nondifferential between groups precludes that these effects on lung function are a result of the phenomenon of regression to the mean [13].

A second methodological issue is related to the duration of the ICS therapy being discontinued. While in the COSMIC and INSTEAD trials, patients had been on ICS for $\geqslant 3$ months, the larger WISDOM trial had given the patients ICS for only 1.5 months, which may be too short a time to observe an effect. Nonetheless, in the subgroup analysis of the $70 \%$ of patients who were on ICS prior to the 1.5 -month ICS run-in period, and had thus used ICS for a longer period, the results on the risk of exacerbation remained similar. As was the case in all these trials, no information was provided on the duration of prior ICS use at the time of randomisation, a key piece of data. A related issue is the increase in treatment due to the trial, such as patients initially on single therapy in WISDOM who end up on double or triple therapy post-randomisation, possibly limiting the effect of withdrawal.

A third issue is that of the study population in terms of the recent history of COPD exacerbation. While in the WISDOM and COSMIC trials patients had to have at least one or two COPD exacerbations, respectively, in the year prior to study entry, the INSTEAD trial selected only patients with no exacerbation during that span, a group that all guidelines would agree should not be on ICS. Interestingly, the two trials (WISDOM and COSMIC) involving the more severe patients (prior exacerbations and

TABLE 2 Two randomised trials of the effect of inhaled corticosteroid (ICS) discontinuation in chronic obstructive pulmonary disease reporting any data on the risk reduction of pneumonia defined as an adverse event

\begin{tabular}{lcccccc}
\multirow{2}{*}{ Trial } & \multicolumn{2}{c}{ ICS discontinuation } & & \multicolumn{2}{c}{ ICS continuation } & Relative risk (95\% CI) \\
\cline { 2 - 3 } & Subjects & Pneumonia & & Subjects & Pneumonia & \\
INSTEAD & 293 & $0(0)$ & & 288 & $2(0.7)$ & $0(0.0$ to 3.4$)$ \\
WISDOM & 1242 & $68(5.5)$ & & 1243 & $72(5.8)$ & $0.95(0.67$ to 1.33$)$ \\
\hline
\end{tabular}

Data are presented as $\mathrm{n}$ or $\mathrm{n}(\%)$, unless otherwise stated. 
baseline predicted FEV1 of $48 \%$ and 34\%) are the two that found significant loss in lung function with ICS discontinuation. The INSTEAD trial, with no prior exacerbations and baseline predicted FEV1 of $64 \%$, found no such effect.

For what should a clinically relevant trial of ICS cessation strive? First, it should select a study population strictly of current users of ICS at randomisation, such as the INSTEAD trial, which should be rather straightforward, as $70 \%$ to $80 \%$ of COPD patients use ICS. Second, a sufficiently long duration of prior continuous ICS use should be imposed to allow detection of the effect of discontinuation. Third, the trial should not restrict on the presence of COPD exacerbations prior to randomisation and be designed to permit stratification by this factor. Finally, the trial should have sufficiently long follow-up to also measure the anticipated benefit from ICS discontinuation in terms of risk reduction, particularly on pneumonia. Indeed, the INSTEAD and WISDOM trials' 6- and 12-month follow-up were probably too short to detect a significant decrease in the rate of serious pneumonia, which also requires some latency.

In conclusion, the use of ICS in COPD has been widespread, even if treatment guidelines have generally limited their place only after one and two long-acting bronchodilators have been initiated. With $>70 \%$ of COPD patients being treated with ICS, the time is ripe to consider the potential effects of weaning many of these patients off the ICS component of their treatment. The studies conducted to date generally suggest that there is no loss of effectiveness on the occurrence of exacerbations when the patients continue exclusively on long-acting bronchodilators, although lung function appears to be reduced, particularly among the patients with more severe disease. Thus, the management of COPD can be improved by limiting the use of ICS to the minority of patients with COPD who benefit, such as patients with the asthma-COPD overlap syndrome and those with more severe disease, and weaning the rest from ICS. More research is needed to determine more clearly the characteristics of the patients who would benefit from ICS discontinuation and to evaluate the impact of such discontinuation on reducing risk, particularly that of pneumonia.

\section{References}

Postma DS, Calverley P. Inhaled corticosteroids in COPD: a case in favour. Eur Respir I 2009; 34: 10-12.

Suissa S, Barnes PJ. Inhaled corticosteroids in COPD: the case against. Eur Respir J 2009; 34: 13-16.

Ernst P, Saad N, Suissa S. Inhaled corticosteroids in COPD: the clinical evidence. Eur Respir J 2015; 45: 525-537.

Vestbo J, Hurd SS, Agusti AG, et al. Global strategy for the diagnosis, management, and prevention of chronic obstructive pulmonary disease: GOLD executive summary. Am J Respir Crit Care Med 2013; 187: 347-365.

5 Corrado A, Rossi A. How far is real life from COPD therapy guidelines? An Italian observational study. Respir Med 2012; 106: 989-997.

6 Roche N, Pribil C, Van GE, et al. Real-life use of fluticasone propionate/salmeterol in patients with chronic obstructive pulmonary disease: a French observational study. BMC Pulm Med 2014; 14: 56.

7 van der Valk P, Monninkhof E, van der Palen J, et al. Effect of discontinuation of inhaled corticosteroids in patients with chronic obstructive pulmonary disease: the COPE study. Am J Respir Crit Care Med 2002; 166: $1358-1363$.

8 Wouters EF, Postma DS, Fokkens B, et al. Withdrawal of fluticasone propionate from combined salmeterol/ fluticasone treatment in patients with COPD causes immediate and sustained disease deterioration: a randomised controlled trial. Thorax 2005; 60: 480-487.

9 Rossi A, van der Molen T, del Olmo R, et al. INSTEAD: a randomised switch trial of indacaterol versus salmeterol/fluticasone in moderate COPD. Eur Respir J 2014; 44: 1548-1556.

10 Magnussen H, Disse B, Rodriguez-Roisin R, et al. Withdrawal of inhaled glucocorticoids and exacerbations of COPD. N Engl J Med 2014; 371: 1285-1294.

11 Suissa S. Statistical treatment of exacerbations in therapeutic trials of chronic obstructive pulmonary disease. $A m \mathrm{~J}$ Respir Crit Care Med 2006; 173: 842-846.

12 Keene ON, Calverley PM, Jones PW, et al. Statistical analysis of exacerbation rates in COPD: TRISTAN and ISOLDE revisited. Eur Respir J 2008; 32: 17-24.

13 Suissa S. Lung function decline in COPD trials: bias from regression to the mean. Eur Respir J 2008; 32: 829-831. 